\title{
Antibodies lock arms against HIV
}

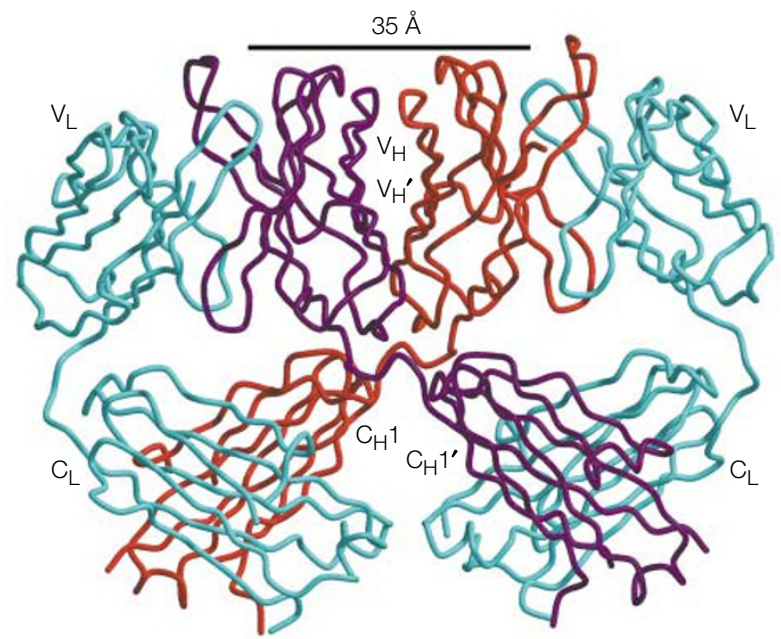

Structure of two domain-swapped Fab molecules. Light chains are shown in blue and the heavy chains from Fab1 and Fab2 are shown in red and purple. Reprinted with permission from Calarese et al. () American Association for the Advancement of Science (2003).
A rapid mutation rate and a protective coating of host-derived sugars seem to have made HIV antibody proof, and in recent years, the neutralizing-antibody approach to HIV vaccine design has taken a back seat. But, neutralizing antibodies are now back in the spotlight, as a recent report in Science shows.

One of the rare neutralizing HIV-specific antibodies that has been isolated from infected patients is the monoclonal antibody $2 \mathrm{G} 12$, which binds with high affinity to oligomannose residues on gp120. To find out exactly how $2 \mathrm{G} 12$ binds to gp120, two groups, led by Dennis Burton and Ian Wilson at the
Scripps Research Institute, La Jolla, USA, determined the crystal structure of the antigen-binding fragment (Fab) of 2G12 together with its carbohydrate ligand. What they discovered was a completely new and unanticipated mode of antigen binding.

Rather than binding the antigen as a monomer, two 2G12 Fabs interlock, forming a dimer in which their heavychain variable $\left(\mathrm{V}_{\mathrm{H}}\right)$ domains are 'swapped'. The result is an extended multivalent antigen-binding site that can recognize the close repeating pattern of sugars that mask the surface of gp120 (see diagram). A conventional antibody could never

INNATE IMMUNITY

\section{DCs: the immune system's flexible friends}

The idea of dendritic-cell (DC) plasticity is gaining momentum, but the ability to secrete large quantities of the type 1 interferons (IFNs), IFN- $\alpha$ and IFN- $\beta$, in response to infection with viruses has long been considered a special feature of plasmacytoid DCs. These cytokines are important for linking innate and adaptive immune responses. But now, Diebold and colleagues report a further level of DC plasticity other types of DC can also produce large quantities of type 1 IFNs.

Because some studies indicated that plasmacytoid DCs are not essential for the production of type 1 IFNs following infection with the Armstrong strain of lymphocytic choriomeningitis virus (LCMV), Diebold and colleagues decided to investigate which other cell types can produce type 1 IFNs. Non-plasmacytoid CD11 chi DCs isolated from mice that were infected with the DCtropic Armstrong variant clone 13 could produce large quantities of type 1 IFNs after in vitro culture.

The authors next investigated the link between virus infection and the production of type 1 IFNs. They used the synthetic double-stranded RNA (dsRNA) mimic polyI:C to stimulate non-plasmacytoid DCs. Mimicking virus infection by cytosolic delivery (by electroporation or delivery with lipofectamine) of polyI:C or virus dsRNA to splenic DCs or bone-marrow-derived DCs enabled them to produce as much type 1 IFN as did plasmacytoid DCs. This was found to be independent of signals delivered through the receptor for dsRNA, Toll-like receptor 3 (TLR3), as the production of IFN- $\alpha$ by bone-marrow-derived DCs from TLR3-deficient mice was unimpaired. Mice deficient in the TLR adaptor MyD88 could also produce IFN- $\alpha$, indicating that production of IFN- $\alpha$ by these DCs is TLR independent.

So, if the TLR pathway isn't important, how is dsRNA recognized by non-plasmacytoid DCs? Protein kinase R (PKR) can bind dsRNA and has been implicated in IFN responses to virus infection. Using PKRdeficient DCs or inhibiting PKR activity led to a reduction in the level of type 1 IFNs, but an increase in the level of interleukin-12, in response to stimulation with polyI:C plus lipofectamine and CD40 ligand.

Many viruses can evade PKR-mediated responses - for example, influenza virus encodes the NS1 protein, which can bind and sequester dsRNA. Does this allow the virus to block the production of type 1 IFNs by nonplasmacytoid DCs? Non-plasmacytoid bonemarrow-derived DCs produce low levels of IFN- $\alpha$ when infected with influenza virus, but infection with an NS1-deficient virus variant resulted in the production of high levels of IFN- $\alpha$, even though this variant infects only a small fraction of bone-marrowderived DCs. Interestingly, influenza virus is often used to stimulate the production of type 1 IFNs by plasmacytoid DCs.

These results show that non-plasmacytoid DCs can produce large amounts of type 1 IFNs during infection with virus and, once again, studying viruses has indicated something new about the immune system a TLR-independent pathway for the activation of DCs.

Elaine Bell

(D) References and links ORIGINAL RESEARCH PAPER Diebold, S. S. et al. Viral infection switches non-plasmacytoid dendritic cells into high interferon producers. Nature 22 June 2003 (DOI: 10.1038/nature01783)

FURTHER READING Banchereau, J. et al. Dendritic cells: controllers of the immune system and a new promise for immunotherapy. Ann. NY Acad. Sci. 987, 180-187 (2003) WEB SITE

Caetano Reis e Sousa's lab: http://science. cancerresearchuk.org/research/loc/london/lifch/sousac/ 